\title{
HAHN DECOMPOSITION FOR THE RIESZ CHARGE OF $\delta$-SUBHARMONIC FUNCTIONS
}

\author{
MIKHAIL SODIN
}

We use standard notions of the potential theory. Let $w$ be a $\delta$-subharmonic function: i.e. a function represented as a difference of two subharmonic functions. Such a function is well defined outside a certain polar set; i.e. quasi-everywhere. For simplicity, we consider functions in the whole complex plane.

Defining a continuous function of $t>0$

$$
t \longmapsto N_{w}(t, z)=\frac{1}{2 \pi} \int_{0}^{2 \pi} w\left(z+t e^{i \theta}\right) d \theta,
$$

we have quasi-everywhere

$$
w(z)=\lim _{t \searrow 0} N_{w}(t, z) .
$$

In fact, that relation can be regarded as a definition of the value $w(z)$ if the limit on the right-hand side exists in $[-\infty, \infty]$. Consider the set $\mathscr{E}_{+}$of points $z$ such that the function $t \mapsto N_{w}(t, z)$ has intervals $\left[r_{n}(z), R_{n}(z)\right]$, $0<r_{n}(z)<R_{n}(z), R_{n}(z) \searrow 0$ as $n \rightarrow \infty$, on which

$$
N_{w}\left(r_{n}, z\right) \leq N_{w}\left(R_{n}, z\right) .
$$

By $\mu[w]$ we denote the Riesz charge of $w(z)$.

THEOREM. The set $\mathscr{E}_{+}$is Borelian, and the restriction $\left.\mu[w]\right|_{\mathscr{E}_{+}}$is a non-negative measure.

\section{Corollaries.}

1. If $w(z) \geq 0$ quasi-everywhere, then

$$
\mathscr{E}_{+} \supset\left\{z: \liminf _{t \backslash 0} N_{w}(t, z)=0\right\} \supset\{z: w(z)=0\},
$$

Received April 11, 1996. 
and we obtain that

$$
\left.\mu[w]\right|_{\{z: w(z)=0\}} \geq 0 .
$$

This statement is known as Grishin's lemma. It was proved in [6], however weaker versions go back to De la Valléé Poussin [11, p.21] and Brelot [1]. It found a number of applications in the potential theory [2], in the subharmonic approach to value-distribution theory [3, 4], in the theory of orthogonal polynomials [9], in complex dynamics [10]. A different proof with further generalizations was given in [5].

2. Let $\mathscr{E}_{-\infty}=\left\{z: \liminf _{t \rightarrow 0} N_{w}(t, z)=-\infty\right\}$. Then $\mathscr{E}_{+} \supset \mathscr{E}_{-\infty}$. Hence

$$
\left.\mu[w]\right|_{\mathscr{E}-\infty} \geq 0 .
$$

3. Let

$$
\mathscr{E}^{*}=\left\{z: \liminf _{t \rightarrow 0} N_{w}(t, z)<\limsup _{t \rightarrow 0} N_{w}(t, z)\right\}
$$

be the polar set of undeterminancy of $w$. Then $\mathscr{E}_{+} \supset \mathscr{E}^{*}$ and $\left.\mu[w]\right|_{\mathscr{E}^{*}} \geq 0$. Replacing $w$ by $-w$, we conclude that

$$
|\mu[w]|\left(\mathscr{E}^{*}\right)=0 .
$$

Corollaries 2 and 3 answer questions posed by Eremenko. Another proof of these two corollaries based on the fine potential theory was given by Fuglede.

4. Define a "dual" set $\mathscr{E}_{-}$of points $z$ such that for certain intervals $\left[r_{n}(z), R_{n}(z)\right], 0<r_{n}(z)<R_{n}(z), R_{n}(z) \searrow 0$ as $n \rightarrow \infty$,

$$
N_{w}\left(r_{n}, z\right) \geq N_{w}\left(R_{n}, z\right) .
$$

Then the domain of the function $w(z)$ splits into three mutually disjoint sets $E_{0}=\mathscr{E}_{+} \cap \mathscr{E}_{-}, E_{+}=\mathscr{E}_{+} \backslash \mathscr{E}_{-}, E_{-}=\mathscr{E}_{-} \backslash \mathscr{E}_{+}$and

$$
\left.\mu[w]\right|_{E_{+}} \geq 0,\left.\quad \mu[w]\right|_{E_{-}} \leq 0,
$$

whilst

$$
|\mu[w]|\left(E_{0}\right)=0 .
$$

Here, $E_{+}$is the set of points $z$ such that the function $t \mapsto N_{w}(t, z)$ increases strictly for $0<t<t_{0}(z)$, and $E_{0}$ is the set of points $z$ such that the function $t \mapsto N_{w}(t, z)$ decreases strictly for $0<t<t_{0}(z)$.

This is the version of the Hahn decomposition of $\mu[w]$ promised in the title of this paper. 


\section{Proof of the Theorem.}

We repeat almost verbatim Grishin's original arguments. First, we prove that $\mathscr{E}_{+}$is a Borelian set. Since the function $z \longmapsto N_{w}(t, z)$ is continuous, the sets $\mathscr{E}(r, R)=\left\{z: N_{w}(R, z) \geq N_{w}(r, z)\right\}$ are closed. In the definition of $\mathscr{E}+$ we may assume without loss of generality that the endpoints $r_{n}, R_{n}$ are rational numbers. Therefore,

$$
\mathscr{E}_{+}=\bigcap_{N=1}^{\infty} \bigcup_{\left\{m, n: \frac{m}{n}<\frac{1}{N}\right\}} \bigcup_{\left\{p, q: \frac{p}{q}<\frac{m}{n}\right\}} \mathscr{E}\left(\frac{p}{q}, \frac{m}{n}\right)
$$

is a Borelian set. Now, let $z \in \mathscr{E}_{+}$. Then by the Jensen formula

$$
\int_{r_{n}}^{R_{n}} \frac{\mu[w](D(z, t))}{t}=N_{w}\left(R_{n}, z\right)-N_{w}\left(r_{n}, z\right) \geq 0,
$$

here, $D(z, t)=\{\zeta:|\zeta-z|<t\}$. Hence, for each $z \in \mathscr{E}_{+}$, there is a sequence of shrinking discs $D\left(z, t_{n}(z)\right), t_{n}(z) \searrow 0$, such that $\mu[w]\left(D\left(z, t_{n}(z)\right)\right) \geq 0$. It remains to apply the following

Claim. Let $E$ be a Borelian set, and let $\nu$ be a charge on the complex plane. Suppose that for each $z \in E$ there is a sequence $t_{n}(z) \searrow 0$ such that

$$
\nu\left(D\left(z, t_{n}(z)\right)\right) \geq 0 .
$$

Then the restriction $\left.\nu\right|_{E}$ is a non-negative measure.

\section{Proof of the Claim.}

Let $F \subset E$ be an arbitrary compact subset. We prove that $\nu(F) \geq 0$ which implies the statement.

First, we choose a decreasing sequence of open sets $O_{j}$ such that $F=\bigcap_{j} O_{j}$ and

$$
\lim _{j}|\nu|\left(O_{j} \backslash F\right)=0 .
$$

Fixing $j$, we assume that all radii $t_{n}(z), z \in F$, are less than the distance from $F$ to $O_{j}$; i.e. all discs $D\left(z, t_{n}(z)\right)$ are contained in $O_{j}$ for $z \in F$.

Now, the generalized Vitali theorem [7, 2.8] gives us a sequence of mutually disjoint discs $D_{n}$ (depending on $j$ ) from the whole collection $\left\{D\left(z, t_{n}(z)\right)\right\}, z \in F, n \in \mathrm{N}$, such that for $G_{j}=\bigcup_{n} D_{n}$

$$
|\nu|\left(F \backslash G_{j}\right)=0 .
$$


Thus $\nu\left(G_{j}\right)=\sum_{n} \nu\left(D_{n}\right) \geq 0$, and

$$
\begin{aligned}
\nu(F) & =\lim _{j} \nu\left(O_{j}\right) \\
& =\lim _{j}\left[\nu\left(O_{j} \backslash\left(G_{j} \cup\left(F \backslash G_{j}\right)\right)\right)+\nu\left(G_{j}\right)+\nu\left(F \backslash G_{j}\right)\right] \geq 0
\end{aligned}
$$

because $|\nu|\left(O_{j} \backslash\left(G_{j} \cup\left(F \backslash G_{j}\right)\right)\right) \leq|\nu|\left(O_{j} \backslash F\right) \rightarrow 0$. We are done.

\section{Remarks.}

1. The assertion of the theorem can be slightly strengthened using the "Phragmén - Lindelöf trick". Let us fix an increasing $C^{1}$-function $h(t)$, $0 \leq t<1, h(0)=0$, such that

$$
h^{\prime}(t)=o(t) \quad \text { as } \quad t \rightarrow 0 .
$$

Then we define a set $\mathscr{E}_{+}^{(h)}$ making use of the function $t \mapsto N_{w}(t, z)+h(t)$ instead of $N_{w}(t, z)$. Evidently, $\mathscr{E}_{+}^{(h)} \supset \mathscr{E}_{+}$. And we still have

$$
\left.\mu[w]\right|_{\mathscr{E}_{+}^{(h)}} \geq 0 .
$$

To prove this we replace $\mu[w]$ by $\mu_{\varepsilon}=\mu[w]+\varepsilon m$, where $m$ is the planararea measure, and $\varepsilon>0$. Then, for $z \in \mathscr{E}_{+}^{(h)}$ and large enough $n$, we have

$$
\begin{aligned}
& \int_{r_{n}}^{R_{n}} \frac{\mu_{\varepsilon}(D(z, t))}{t} d t \\
& =N_{w}\left(R_{n}, z\right)-N_{w}\left(r_{n}, z\right)+\frac{\pi}{2} \varepsilon\left(R_{n}^{2}-r_{n}^{2}\right) \\
& \geq\left[N_{w}\left(R_{n}, z\right)+h\left(R_{n}\right)\right]-\left[N_{w}\left(r_{n}, z\right)+h\left(r_{n}\right)\right] \geq 0 .
\end{aligned}
$$

(in the first inequality we have used the estimate

$$
h\left(R_{n}\right)-h\left(r_{n}\right)=\int_{r_{n}}^{R_{n}} h^{\prime}(t) d t \leq \pi \varepsilon \int_{r_{n}}^{R_{n}} t d t=\frac{\pi \varepsilon}{2}\left[R_{n}^{2}-r_{n}^{2}\right]
$$

if $n$ is large enough).

Now, using the Claim we conclude that $\mu_{\varepsilon_{\mathscr{E}_{+}}} \geq 0$ and since $\varepsilon$ is an arbitrary number $\left.\mu[w]\right|_{\mathscr{E}_{+}^{(h)}} \geq 0$.

2. One may go a little bit further in this direction. Let $E$ be a Borelian set. Suppose that for some $s>0$ there is a non-negative measure $\lambda_{s}$ such that for each $z \in E$ and each $t, 0<t<t_{0}(z)$,

$$
\lambda_{s}(D(z, t)) \geq t^{s} .
$$

The existence of such a measure is provided by the so-called "anti Frostman lemma" proved in [8, Lemma 4] for 


$$
s>\Delta(E)=\limsup _{\varepsilon \rightarrow 0} \frac{\log M_{\epsilon}(E)}{\log 1 / \varepsilon},
$$

where $M_{\varepsilon}(E)$ is the smallest number of discs of radius $\varepsilon$ required to cover $E$. The value $\Delta(E)$ is called the upper Minkowski dimension or the box counting dimension. In general, it is bigger than or equal the Hausdorff dimension, however for many "reasonable" sets these two dimensions coincide. For the discussion of this concept see [7, Chapter 5] and [8]. Ifsuch $\lambda_{s}$ exists, we can consider the intersection $\mathscr{E}_{+}^{(h)} \cap E$ where $\mathscr{E}_{+}^{(h)}$ is defined with an increasing $h(t), h(0)=0$, which is $C^{1}$ and such that

$$
h^{\prime}(t)=o\left(t^{s-1}\right), \quad t \rightarrow 0 .
$$

The same argument as above with $\mu_{\varepsilon}=\mu[w]+\varepsilon \lambda_{s}, \varepsilon>0$, shows that

$$
\left.\mu[w]\right|_{\mathscr{E}_{+}^{(h)} \cap E} \geq 0 .
$$

Acknowledgements. I thank Alexandre Eremenko, Bent Fuglede, Pertti Mattila and Flemming Topsøe for useful discussions. I also thank the referee for many useful remarks. The first version of this paper was written during my stay at the Mathematical Institute, University of Copenhagen as a Guest lecturer in the academic year 1994/95. I am most grateful to this institution and the colleagues for generous hospitality. This work was done in the framework of the INTAS 94-1474 research network "Potential theory, complex analysis and their applications".

\section{REFERENCES}

1. M. Brelot, Sur l'allure des fonctions harmoniques etsousharmoniques à la frontière, Math. Nachr. 4 (1950/51), 298-307.

2. A. Eremenko, B. Fuglede and M. Sodin On the Riesz charge of the lower envelope of $\delta$-subharmonic functions, Potential Analysis 1 (1992), 191-204.

3. A. Eremenko and M. Sodin, The value distribution of meromorphic functionsand meromorphic curves from the point of view of potential theory, St. Petersburg Math. J. 3 (1992), 109-136.

4. A. Eremenko and M. Sodin, On the distribution of values of meromorphicfunctions of finite order, Soviet Math. Dokl. 43 (1991), 128-131.

5. B. Fuglede, Some properties of the Riesz charge associatedwith a $\delta$-subharmonic function, Potential Analysis 1 (1992), 355-371.

6. A. Grishin, Sets of regular growth of entire functions, I, Teor. Funktsii, Funktsional. Anal. i Prilozhen (Kharkov) 40 (1983), 36-47 (in Russian).

7. P. Mattila, Geometry of Sets and Measures, Cambridge University Press, Cambridge, 1995.

8. C. Tricot, Two definitions of fractional dimension, Math. Proc. Cambridge. Philos. Soc. 91 (1982), 57-74. 
9. V. Totik and J. L. Ullman, Local asymptotic distribution of zeros of orthogonal polynomials, Trans. Amer. Math. Soc. 381 (1994), 881-894.

10. A. Volberg, Colloquium talk at the Mathematical Institute,University of Copenhagen, June 1995.

11. C. De la Vallée Poussin, Potential et problème généralisé de Dirichlet, Math. Gazette, London 22 (1938), 17-36.

MATHEMATICAL DIVISION

INSTITUTE FOR LOW TEMPERATURE PHYSICS

LENIN'S PR. 47

KHARKOV 310164

UKRAINE
SCHOOL OF MATHEMATICAL SCIENCES TEL-AVIV UNIVERSITY

RAMAT-AVIV, 69978

ISRAEL 